\title{
Resistência específica à tração na operação de escarificação do solo em camadas de forma simultânea
}

\author{
José R. F. Gassen ${ }^{1}$, Airton dos S. Alonço², Ulisses B. Baumhardt', \\ Mateus P. Bellé \& Gustavo J. Bonotto ${ }^{5}$ \\ ${ }^{1}$ PPGEA/UFSM - IFMT, Cuiabá, MT. E-mail: jrgassen@gmail.com (Autor correspondente) \\ 2 PPGEA/UFSM, Santa Maria, RS. E-mail: airtonalonco@gmail.com \\ ${ }^{3}$ UFSM/PPGEA - UNIPAMPA, Bagé, RS. E-mail: ulissesbb@brturbo.com.br \\ ${ }^{4}$ PPGEA/UFSM, Santa Maria, RS. E-mail: mateuspotrichbelle@yahoo.com.br \\ ${ }^{5}$ PPGEA/UFSM, Santa Maria, RS. E-mail: gustavobonotto@yahoo.com.br
}

\section{Palavras-chave:}

preparo conservacionista do solo projeto de máquinas agrícolas

compactação do solo

escarificador

força de tração

\begin{abstract}
R E S U M O
O aumento do tráfego de máquinas agrícolas maiores e mais robustas agrava a compactação do solo afetando, direta e indiretamente, a produtividade das culturas. Para solucionar este problema tem-se realizado a escarificação; contudo, esta operação apresenta elevada demanda de tração. Neste contexto e visando a uma operação de escarificação mais eficiente, desenvolveu-se e se avaliou uma ferramenta para o rompimento do solo em camadas, de forma simultânea. O experimento foi conduzido no Município de Capão Bonito do Sul, Rio Grande do Sul, em um solo de textura muito argilosa. Como objetivo geral buscou-se verificar se existe diferença significativa entre a escarificação em única camada ou convencional, e em mais de uma camada, de forma simultânea, considerando-se a resistência específica como critério para três níveis de velocidade. Foram testadas configurações com escarificação do solo em uma, duas e três camadas. Em duas camadas simultâneas de $15 \mathrm{~cm}$ e distância longitudinal entre as pontas das ponteiras, tanto de 30 quanto de $40 \mathrm{~cm}$ observou-se que a escarificação e aquela realizada em camadas de $10 \mathrm{e}$ avanço de $30 \mathrm{~cm}$, se apresentaram mais promissoras.
\end{abstract}

\section{Key words:}

soil conservation tillage

design of agricultural machinery

soil compaction

chisel plow

traction force

\section{Specific resistance to traction in the operation of simultaneous chiseling of the soil layers}

\begin{abstract}
A B S T R A C T
The increased traffic of larger and more robust agricultural machinery, worsen the soil compaction, affecting directly and indirectly crop productivity. To solve this problem the chiseling has been used; however, this operation has a high demand for traction. In this context, seeking a more efficient operation of chiseling, an implement was developed and evaluated to break simultaneously the soil layers. The experiment was carried out in the municipality of Capão Bonito do Sul, Rio Grande do Sul, in a clayey soil. The general objective was to determine whether there is any significant difference between chiseling in a single layer or in more than one layer while considering the specific resistance as a criterion for three levels of speed. Configurations were tested with chiseling of the soil in one, two and three layers. It was concluded that the chiseling of two layers simultaneously of $15 \mathrm{~cm}$ and the longitudinal distance between the tip ends of $30 \mathrm{~cm}$ as well as of $40 \mathrm{~cm}$, together with that one performed in layers of $10 \mathrm{~cm}$ and advance of $30 \mathrm{~cm}$ presented as the most promising.
\end{abstract}

\section{INTRODUÇÃ̃o}

Vem-se observando, nos últimos anos, uma intensificação na busca por soluções agrícolas que visem à preservação do meio ambiente e à conservação do solo, o que se revela como grande desafio face à necessidade de altas produtividades das culturas e racionalização dos custos de produção. Uma das consequências desta necessidade é a crescente demanda de energia associada à intensificação do uso de máquinas agrícolas mais robustas e maiores, devido às exigências do sistema de semeadura direta afetando, de forma significativa, a compactação do solo a qual atua direta e indiretamente de modo negativo sobre a produtividade das culturas visto que modifica diversos atributos físicos do solo, entre os quais se destacam: densidade, resistência à penetração, macro e microporosidade e capacidade de retenção de água.

A compactação do solo é um processo em que a porosidade e a permeabilidade são reduzidas, a resistência mecânica é aumentada e muitas mudanças ocorrem na estrutura do solo (Soane \& Ouwerkerk, 1994). Segundo Flowers \& Lal (1998), a principal causa da compactação em solos são as condições de tráfego das máquinas usadas nas operações agrícolas, tais como 
operações de preparo, semeadura, tratos culturais e colheita. Para Drescher et al. (2011), este adensamento do solo nas áreas agrícolas pode interferir diretamente no desempenho de máquinas e implementos agrícolas promovendo uma ampliação na demanda de potência para tração. Foloni et al. (2003) concluíram, em estudo sobre o efeito da compactação do solo no desenvolvimento aéreo e radicular de cultivares de milho, que: (i) a compactação do solo comprometeu o desenvolvimento das plantas de milho híbrido e da variedade na mesma intensidade; (ii) apesar de alterar a distribuição do sistema radicular ao longo do perfil do solo, o impedimento físico em subsuperfície não diminuiu a produção total de raízes de milho; (iii) o diâmetro médio radicular apresentou alta correlação com o crescimento de raízes no solo compactado e (iv) o sistema radicular do milho não é capaz de romper uma camada compactada de solo com resistência mecânica da ordem de 1,4 MPa. Beutler \& Centurion (2004) verificaram, ao pesquisar o efeito da compactação do solo no desenvolvimento radicular e na produtividade da soja, em Latossolo Vermelho de textura média, que houve diminuição na produtividade e em determinada profundidade ocorreu uma redução na densidade das raízes.

Collares et al. (2006) encontraram, em experimento na cultura do feijoeiro, influência direta da compactação na redução da produtividade desta cultura enquanto Beutler et al. (2004) obtiveram, avaliando a influência da compactação na produtividade e altura do sistema radicular em arroz de sequeiro, redução na produtividade e restrição ao crescimento das raízes. Como técnica potencial para solucionar este problema tem-se a escarificação que promove o rompimento dessas camadas compactadas ou adensadas. Para Machado et al. (2005), a operação de escarificação consiste em mobilizar o solo a determinada profundidade até trinta centímetros, tendo uma mobilização superficial mínima e mantendo a cobertura do solo. Por não provocar inversão de camadas do solo, esta operação proporciona menor desagregação sendo que os resíduos vegetais ficam depositados na superfície do solo facilitando, assim, o controle da erosão, melhorando potencialmente a infiltração e a retenção de água, tal como a estrutura e a porosidade do solo, quando comparada aos preparos do solo convencionais. Esses mesmos autores, ao mencionar a parte ativa do escarificador (as ponteiras) a divide em dois tipos, estreita, com largura de 4 a 8 $\mathrm{cm}$ e larga ou alada, com dimensões acima destas. Nicoloso et al. (2008) consideraram a escarificação mecânica como alternativa eficiente para melhorar as condições físicas do Latossolo de textura muito argilosa quando associada à escarificação biológica auxiliando na prevenção da reconsolidação do solo em experimento de campo realizado em quatro áreas, no município de Santa Rosa, região Noroeste do Rio Grande do Sul. Na busca de uma operação mais eficiente, Godwin (2007) destaca que a busca pela redução da demanda de tração de um implemento não deve ser o objetivo mais importante mas sim reduzir a resistência específica operacional expressa pela relação da força de tração com a área de solo mobilizado.

Assim, objetivou-se neste trabalho, desenvolver e analisar uma ferramenta para o rompimento do solo em camadas simultâneas, ou seja, se a profundidade de trabalho de um escarificador tem influência significativa no desempenho do implemento então é possível romper o solo em camadas de modo simultâneo, de forma que a eficiência do equipamento melhore, alcançando menor resistência específica para cada velocidade de trabalho testada.

\section{MATerial e Métodos}

A área experimental utilizada está situada no município de Capão Bonito do Sul, localizado na região dos Campos de Cima da Serra, na porção nordeste do estado do Rio Grande do Sul. Tal região é constituída de um relevo plano com cotas próximas a $800 \mathrm{~m}$ de altitude.

A localização da área possui Latitude $28^{\circ} 09^{\prime} 2.93^{\prime \prime} \mathrm{S}$ e Longitude $51^{\circ} 29^{\prime} 13.72^{\prime}$. A inclinação média na direção das linhas de tração foi de 5,60\% (declive) e na transversal de $6,10 \%$ (declive). A área é utilizada há cinco anos sob o sistema de plantio direto com cultivo de milho para ensilagem, não havendo descompactação do solo há, pelo menos, oito anos. O trabalho foi realizado após a ensilagem do milho havendo uma mínima quantidade de resíduo vegetal na superfície e com tráfego intenso de máquinas, situação que evidenciou a existência de compactação diagnosticada após a determinação dos valores da resistência à penetração na malha composta por trinta e seis pontos, os pontos amostrados foram distribuídos na área experimental, sendo oito amostragens por bloco.

Os valores medidos de resistência à penetração e densidade do solo estão apresentados em forma de resumo, na Tabela 1. Analisada a densidade aparente do solo, observa-se uma leve tendência de maior compactação nos dois primeiros intervalos de profundidade, em todos os blocos. Ao observar a Tabela 1 nota-se tendência geral de menor resistência à penetração média na profundidade de $20 \mathrm{~cm}$ e maior compactação a 10 $\mathrm{cm}$. Conforme Foloni et al. (2003), uma camada compactada com resistência à penetração de $1400 \mathrm{kPa}$ impede que o sistema radicular do milho a atravesse e se desenvolva em profundidade. Pode-se observar, também, que o comportamento é diferenciado dentro de cada bloco enquanto no bloco um a ordem decrescente de compactação é P2', P1; P2; P3, no bloco dois é P3, P2'; P1; P3, no três P1; P3; P2'; P2 e por fim no quatro é P1; P2', P3; P2. Esta tendência evidencia potencialmente um perfil de solo com duas camadas mais compactadas acima (P1 e P2') separadas de outra (P3), por uma camada menos compactada (P2).

Tabela 1. Resistência à penetração média e densidade do solo em função da profundidade

\begin{tabular}{lccccc}
\hline & \multicolumn{4}{c}{ Blocos } & \multirow{2}{*}{ Média } \\
\cline { 2 - 5 } & I & II & III & IV & \\
Resistência à penetração - kPa & & & & \\
RP 0,10 m - (P1) & 1.563 & 1.500 & 1.528 & 1.706 & 1.574 \\
RP 0,15 m - (P2') & 1.587 & 1.581 & 1.522 & 1.422 & 1.528 \\
RP 0,20 m - (P2) & 1.508 & 1.428 & 1.297 & 1.413 & 1.411 \\
RP 0,30 m - (P3) & 1.459 & 1.597 & 1.474 & 1.466 & 1.499 \\
\hline Densidade do solo - Mg m m $^{-3}$ & & & & \\
Ds 0,00-0,10 m (P1) & 1,28 & 1,36 & 1,34 & 1,35 & 1,33 \\
Ds 0,10-0,20 m (P2) & 1,32 & 1,40 & 1,29 & 1,38 & 1,35 \\
Ds 0,20-0,30 m (P3) & 1,17 & 1,22 & 1,29 & 1,28 & 1,24 \\
\hline
\end{tabular}


O teor de água do solo no dia do experimento era, em média, de $22 \%$, o limite de plasticidade $42 \%$ e o de liquidez de $53 \%$.

O solo desta região é classificado como Latossolo Vermelho Aluminoférrico típico. Observando a análise granulométrica do solo, na Tabela 2, nota-se que se trata de um solo de textura muito argilosa.

Tabela 2. Análise granulométrica do solo e densidade de partículas (Dp)

\begin{tabular}{ccccc}
\hline Camadas & \multicolumn{3}{c}{ Granulometria (\%) } & Dp \\
\cline { 2 - 4 }$(\mathbf{m})$ & Areia & Silte & Argila & $\left(\mathbf{M g ~ m}^{-3}\right)$ \\
$0-0,10$ & 3,3 & 34,2 & 62,5 & 2,73 \\
$0,10-0,20$ & 3,2 & 33,6 & 63,2 & 2,78 \\
$0,20-0,30$ & 3,0 & 31,3 & 65,7 & 2,75 \\
\hline
\end{tabular}

Quanto à porosidade total e à microporosidade, a última compôs, praticamente, $90 \%$ da primeira indicando que, na verdade, o solo se encontrava compactado, visto que deveria existir um equilíbrio entre a macro e a microporosidade.

Para determinação da textura e densidade dos sólidos foram retirados dois grupos de três amostras estratificadas por bloco (0-10, 10-20 e 20-30 cm). Essas amostras foram misturadas obedecendo às faixas de mesma profundidade para, ao final, se retirar três amostras finais de cada intervalo de profundidade. Deste material também foi retirada certa quantidade para determinação dos limites de liquidez e plasticidade e cálculo do índice de plasticidade. Na determinação da densidade aparente da microporosidade e do teor de água do solo foram retirados dois grupos de três amostras estratificadas por bloco (0-10, 10 -20 e $20-30 \mathrm{~cm}$ ), as quais foram impermeabilizadas, pesadas e levadas ao laboratório para secagem e pesagem posterior.

Como fonte móvel de potência foi usado um trator de rodas $4 \mathrm{x} 2$ simples com potência no motor de $53 \mathrm{~kW}$, marca Massey Ferguson, modelo 275, ano de fabricação 1986. Tal trator foi equipado com uma instrumentação eletrônica, a qual foi baseada em Russini (2009). Utilizou-se uma Estrutura Porta-Ferramentas Móvel (EPFM) hastes e ponteiras que foram projetadas, construídas e montadas no LASERG em parceria com a Stara Indústria de Implementos Agrícolas S.A. Como se visualiza na Figura 1, a EPFM possui um chassi fixo, em verde, para acoplamento das hastes com possibilidade de regulagem de altura em relação ao solo e sistema de levante, em azul, que

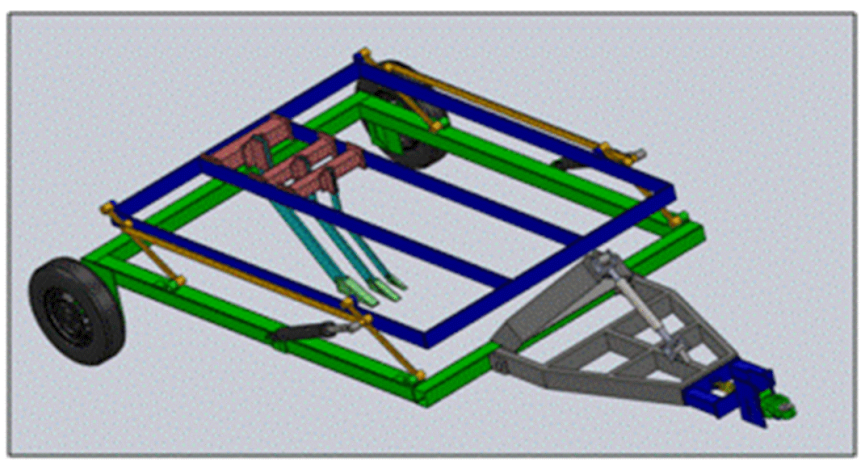

Figura 1. Estrutura porta-ferramentas móvel, hastes e ponteiras permite a penetração do órgão ativo na posição e ângulo ideais. Ela é acoplada na barra de tração do trator e a sustentação de sua massa se dá por três pontos, ou seja, um apoio na barra de tração, em cinza, e duas rodas situadas na parte traseira do implemento, em cor preta.

A instrumentação utilizada foi composta por uma célula de carga para a aferição da força horizontal, com capacidade de 5 toneladas, marca Alfa equipamentos, um GPS para captar a velocidade de deslocamento, marca Garmin, modelo MAP60CS, dois sensores indutivos acoplados aos rodados motrizes para suas velocidades e após o cálculo do patinamento, modelo LM 12-3004 PC, um datalogger para o armazenamento dos dados, marca Campbell Scientific, modelo CR-1000, um penetrômetro digital para o conhecimento da resistência à penetração, marca Falker, modelo PLG 1020 com ponteira n 2 e um perfilômetro para aferição do relevo original, perfil empolado e perfil basal do solo.

Quanto ao perfilômetro, apresentado na Figura 2, construído em estrutura de aço com 0,60 $\mathrm{m}$ de largura, composto por quinze hastes em alumínio (barras circulares) com diâmetro de 0,012 $\mathrm{m}$ e comprimento $0,55 \mathrm{~m}$, espaçadas $0,052 \mathrm{~m}$, possuem uma placa de madeira removível na qual foram fixadas as folhas de papel milimetrado A2. A capacidade de medição é de até 0,35 $\mathrm{m}$ de profundidade.

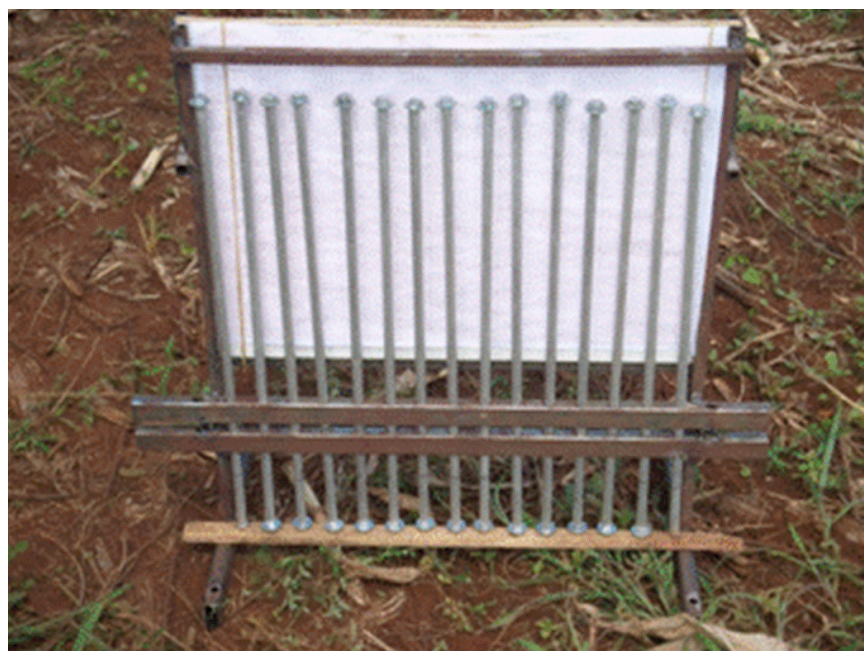

Figura 2. Perfilômetro de hastes

Para pesagem das amostras, tanto no campo quanto no laboratório, foi usada uma balança digital com 0,001 g de resolução. $\mathrm{Na}$ secagem das amostras foi utilizada uma estufa elétrica regulada em $105 \pm 5{ }^{\circ} \mathrm{C}$. As determinações da macro e microporosidade, densidade dos sólidos, granulometria e outros testes de mecânica do solo, como limites de Atterberg, foram realizados no Laboratório de Física dos Solos da UFSM de acordo com a EMBRAPA (1997).

A ferramenta desenvolvida e estudada é apresentada na Figura 3, juntamente com a identificação dos parâmetros característicos. Considerando esta figura observam-se três fatores de influência: "Pt" - profundidade de trabalho -, "Ap" - aprofundamento - e "Av" - avanço da ponteira. O 


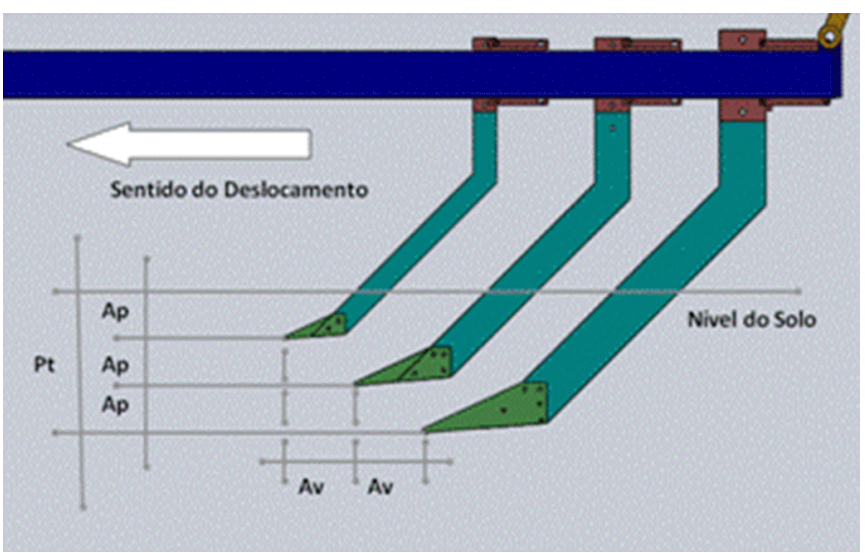

Figura 3. Parâmetros relativos à ferramenta para escarificação progressiva

aprofundamento é a espessura da camada de solo a ser rompida por cada uma das hastes e o avanço da ponteira é a distância longitudinal entre as pontas das ponteiras (frentes de rompimento).

Com relação ao experimento de campo durante os tratamentos, além das variáveis resposta principais, força horizontal média na barra de tração (FHMBT), área de solo mobilizado (Asm) e resistência específica $\left(\mathrm{R}_{\text {esp }}\right)$, também foram quantificadas a resistência à penetração do solo (RP) e a patinagem do trator $(\mathrm{Pi})$. O delineamento experimental utilizado foi blocos ao acaso.

O experimento teve por objetivo avaliar os efeitos decorrentes de três fatores contra um tratamento testemunha, com quatro repetições, num Latossolo Vermelho Distrófico alumino férrico, totalizando oitenta e quatro parcelas:

(i) Fator 1 - Aprofundamento ou espessura da camada com dois níveis (Ap1 $=10 \mathrm{~cm}-3$ hastes; Ap2 $=15 \mathrm{~cm}-2$ hastes) e uma testemunha ( $\mathrm{Ap} 0=\mathrm{Pt}=30 \mathrm{~cm}-1$ haste);

(ii) Fator 2 - Avanço ou distância longitudinal entre as pontas das ponteiras, com três níveis $(\mathrm{Av} 1=40 \mathrm{~cm}$; Av2 $=30$ $\mathrm{cm} ; \mathrm{Av} 3=20 \mathrm{~cm}$ ); e

(iii) Fator 3 - Velocidade de trabalho com três níveis $(\mathrm{V} 1=$ $3,0 \mathrm{~km} \mathrm{~h}^{-1} ; \mathrm{V} 2=6,0 \mathrm{~km} \mathrm{~h}^{-1} ; \mathrm{V} 3=7,0 \mathrm{~km} \mathrm{~h}^{-1}$ ).

Cada parcela foi dimensionada com $2,5 \mathrm{~m}$ de largura por $25 \mathrm{~m}$ de comprimento sendo que os primeiros e os últimos $2,5 \mathrm{~m}$, serviram para estabilização da velocidade durante a entrada e saída da ferramenta no solo. As velocidades adotadas no experimento foram $3,6 \mathrm{e} 7 \mathrm{~km} \mathrm{~h}^{-1}$ e as marchas, respectivamente, segunda reduzida, terceira reduzida e quarta reduzida; estas foram alcançadas através de procedimento no qual foi especificada uma rotação de $1900 \mathrm{rpm}$ no motor do trator seguida da busca por uma marcha adequada.

Como sinalizado na Tabela 3, foram três os tratamentos testemunha, nos quais as ponteiras utilizadas foram do tipo estreito, com largura de aproximadamente um quinto da profundidade de trabalho $(\mathrm{Pt}=30 \mathrm{~cm}$ de profundidade de trabalho), com três diferentes velocidades. Além desses também foram montadas e ensaiadas dezoito combinações de configurações de hastes e velocidades. Os nove primeiros com
Tabela 3. Caracterização dos tratamentos executados nos experimentos de campo

\begin{tabular}{|c|c|c|c|}
\hline \multirow{2}{*}{ Tratamentos } & Aprofundamento & Avanço & \multirow{2}{*}{$\begin{array}{l}\text { Velocidade de } \\
\text { trabalho }\left(\mathrm{km} \mathrm{h}^{-1}\right)\end{array}$} \\
\hline & (cm) & & \\
\hline T01 & $A p_{0}=P_{t}=30$ & ---- & $V_{1}=3,0$ \\
\hline T02 & $A p_{0}=P_{t}=30$ & --- & $V_{2}=6,0$ \\
\hline T03 & $A p_{0}=P_{t}=30$ & ---- & $V_{3}=7,0$ \\
\hline $\mathrm{T} 1$ & $A p_{1}=10 \mathrm{~cm}$ (3 hastes) & $A v_{1}=40$ & $V_{1}=3,0$ \\
\hline $\mathrm{T} 2$ & $A p_{1}=10$ & $A v_{1}=40$ & $V_{2}=6,0$ \\
\hline T3 & $A p_{1}=10$ & $A v_{1}=40$ & $V_{3}=7,0$ \\
\hline $\mathrm{T} 4$ & $A p_{1}=10$ & $A v_{2}=30$ & $V_{1}=3,0$ \\
\hline T5 & $A p_{1}=10$ & $A v_{2}=30$ & $V_{2}=6,0$ \\
\hline T6 & $A p_{1}=10$ & $A v_{2}=30$ & $V_{3}=7,0$ \\
\hline $\mathrm{T} 7$ & $A p_{1}=10$ & $A v_{3}=20$ & $V_{1}=3,0$ \\
\hline T8 & $A p_{1}=10$ & $A v_{3}=20$ & $V_{2}=6,0$ \\
\hline T9 & $A p_{1}=10$ & $\mathrm{Av}_{3}=20$ & $V_{3}=7,0$ \\
\hline T10 & $\mathrm{Ap}_{2}=15 \mathrm{~cm}(2$ hastes $)$ & $A v_{1}=40$ & $V_{1}=3,0$ \\
\hline T11 & $\quad A p_{2}=15$ & $A v_{1}=40$ & $V_{2}=6,0$ \\
\hline $\mathrm{T} 12$ & $A p_{2}=15$ & $A v_{1}=40$ & $V_{3}=7,0$ \\
\hline T13 & $A p_{2}=15$ & $\mathrm{Av}_{2}=30$ & $V_{1}=3,0$ \\
\hline $\mathrm{T} 14$ & $A p_{2}=15$ & $A v_{2}=30$ & $V_{2}=6,0$ \\
\hline T15 & $A p_{2}=15$ & $\mathrm{Av}_{2}=30$ & $V_{3}=7,0$ \\
\hline T16 & $A p_{2}=15$ & $A v_{3}=20$ & $V_{1}=3,0$ \\
\hline T17 & $A p_{2}=15$ & $A v_{3}=20$ & $V_{2}=6,0$ \\
\hline T18 & $A p_{2}=15$ & $A v_{3}=20$ & $V_{3}=7,0$ \\
\hline
\end{tabular}

rompimento do solo em três camadas aproximadamente iguais $\left(\mathrm{Ap} 1=\mathrm{Pt}^{-1}\right)$ e três avanços decrescentes $(\mathrm{Av} 1=40 \mathrm{~cm}$; Av2 $=$ $30 \mathrm{~cm}$ e Av3 $=20 \mathrm{~cm}$ ), enquanto nos nove restantes se tem duas camadas aproximadamente iguais $(\mathrm{Ap} 2=\mathrm{Pt} / 2)$ e os mesmos três avanços decrescentes $(\mathrm{Av} 1=40 \mathrm{~cm} ; \mathrm{Av} 2=30 \mathrm{~cm}$ e Av3 $=20$ $\mathrm{cm})$. A nomenclatura usada para os tratamentos com o objetivo de romper o solo em camadas é composta por TX (Ap; Av; V), donde " $\mathrm{T}$ " significa tratamento, "X" identifica a ordem deste variando de 1 a 18 e os termos entre parênteses da esquerda para a direita são: Aprofundamento (espessura de cada camada), Avanço (distância longitudinal entre as pontas das ponteiras ou frentes de rompimento) e velocidade da operação. Visando à identificação dos tratamentos testemunhas ou controles, a nomenclatura recebe um zero entre a letra " $\mathrm{T}$ " e o número de ordem "Y", que varia de 1 a 3 , T0Y $(30 ; 00 ; \mathrm{V})$.

$\mathrm{A} \mathrm{F}_{\mathrm{HMBT}}$ foi calculada como a diferença entre a média da força de tração medida durante o experimento $\left(\mathrm{F}_{\mathrm{HBtMed}}\right)$ e a média da resistência ao rolamento do conjunto $\left(\mathrm{R}_{\mathrm{ROL}}\right)$ conforme Eq. 1:

$$
\mathrm{F}_{\mathrm{HMBT}}=\mathrm{F}_{\mathrm{HBTMed}}-\mathrm{R}_{\mathrm{ROL}}
$$

A média da força medida na barra de tração a ser considerada na análise é a média das forças instantâneas medidas nos $25 \mathrm{~m}$ da unidade experimental, coletadas e armazenadas automaticamente pela instrumentação a cada 2 s. A resistência ao rolamento $\left(\mathrm{R}_{\mathrm{ROL}}\right)$ foi determinada pela média dos valores medidos de força horizontal média na barra de tração necessária para tracionar o conjunto estrutura porta-ferramentas móvel e ferramentas não enterradas no solo. Para determinação da área de solo mobilizado foi instalado um perfilômetro na linha média de cada unidade experimental anterior à passagem da ferramenta no sentido transversal ao deslocamento do conjunto 
(trator + estrutura porta-ferramentas) determinando, desta forma, o microrrelevo do solo mobilizado. Na sequência e imediatamente à passagem da ferramenta nesta mesma seção transversal, foi reinstalado o mesmo instrumento de medição e obtido o empolamento do solo. Por fim, após remoção completa do solo desagregado foi determinado o perfil basal do sulco. Com esses dados e um tratamento adequado gráfico/matemático, determinaram-se as áreas em questão. Tal tratamento de dados consistiu em inseri-los em uma planilha eletrônica e nesta, através da utilização de fórmulas para cálculo de áreas, obtiveram-se os resultados procurados. Visando à conferência dessas áreas plotaramse, em sistema $\mathrm{CAD}$, os pontos referentes às leituras do

A.

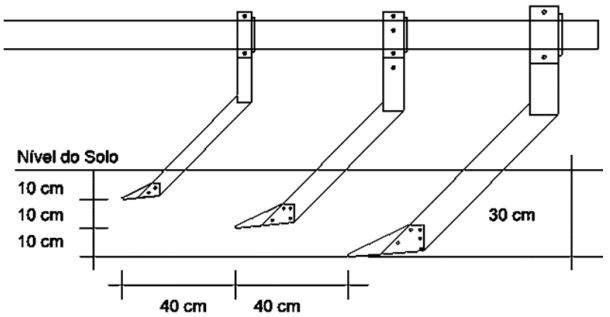

B.

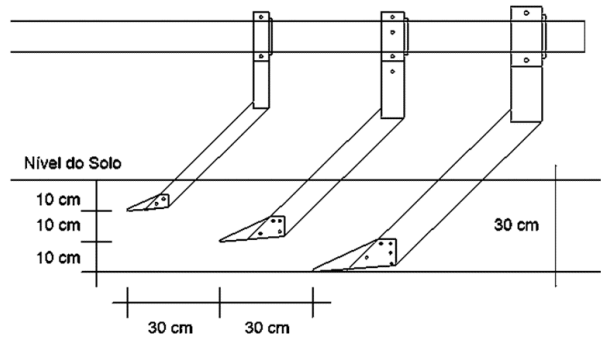

C.

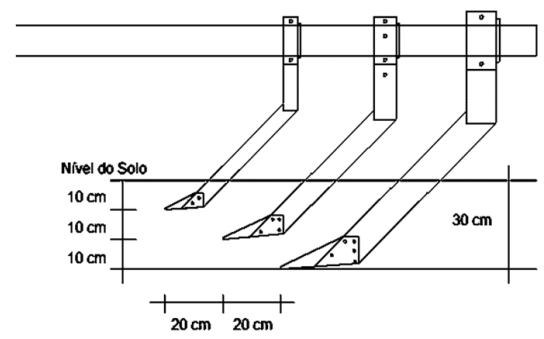

perfilômetro e, por recursos do sistema, determinaram-se algumas áreas, escolhidas ao acaso, comparando-as com as calculadas. Relacionando a força horizontal média na barra de tração com a área de solo mobilizada tem-se a Resistência Específica dada em $\mathrm{kN} \mathrm{m}^{-2}$, a qual foi o critério usado na análise estatística visto que esta é um indicativo do dispêndio de energia por unidade de solo descompactado.

$\mathrm{Na}$ sequência tem-se a apresentação da Figura 4, composta pelos esquemas representativos das configurações utilizadas no teste de campo.

Os tratamentos propostos visavam responder se existe alguma diferença significativa entre a escarificação em única camada em comparação àquela em mais de uma camada de

D.

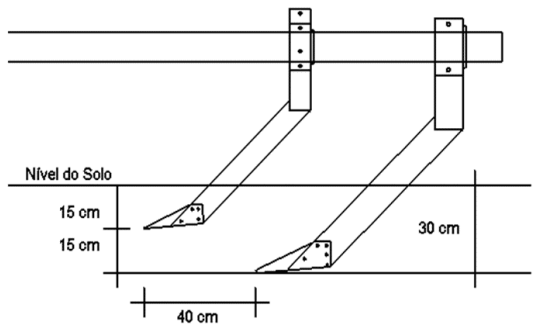

E.

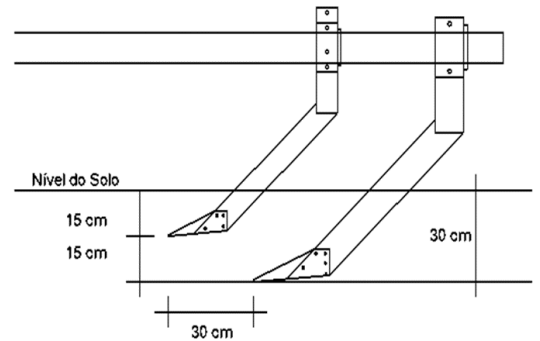

F.

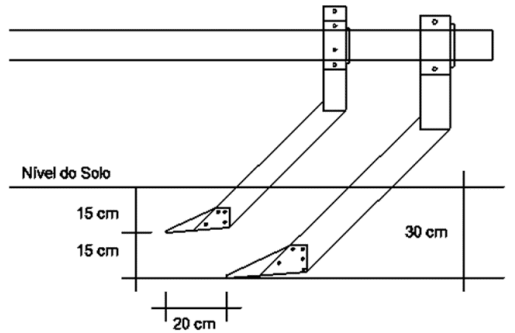

G.

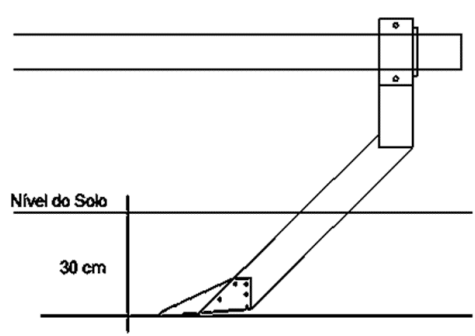

Figura 4. Esquemas representativos das configurações utilizadas no teste de campo: (A) configuração de hastes que rompe o solo em três camadas de $10 \mathrm{~cm}$ de espessura com a distância longitudinal entre as pontas das ponteiras de $40 \mathrm{~cm}$; (B e C), o número de camadas permanece o mesmo mas o avanço entre as pontas diminui para 30 e $20 \mathrm{~cm}$, respectivamente; na coluna da direita são mostrados os esquemas de montagem das hastes para o rompimento do solo em duas camadas, primeiramente (D) com avanço de 40 decrescendo para 30 (E) e $20 \mathrm{~cm}$ (F) e está representada a configuração usada para os tratamentos testemunhas ou controles com uma haste operando na profundidade de trabalho, de modo a romper o solo em uma única camada $(\mathrm{G})$ 
forma simultânea, considerando-se a resistência específica como critério, para cada nível de velocidade. Para tanto foram comparados os tratamentos testemunha com as configurações de hastes para cada nível de velocidade, através do software Assistat Versão 7.6 beta (Silva \& Azevedo, 2002), em experimentos fatoriais diferenciados pelo teste de Dunnett. Segundo Banzatto \& Kronka (2008), este teste é usado quando interessam, ao experimentador, as comparações entre determinado tratamento padrão (testemunha) e cada um dos demais, seus resultados se apresentam por barras verticais, de forma que quando estas estão alinhadas as médias dos tratamentos testados não diferem estatisticamente daquela relativa ao controle. Os fatores, mesmo sendo expressos por números, nesta análise são considerados qualitativos visto que os mesmos se apresentam com poucos níveis: o aprofundamento possui dois: o avanço três e a velocidade da operação também possui três níveis. Além disto, não faz parte deste trabalho a obtenção de modelos ajustados para a predição de força de tração, resistência específica e consumo de combustível, dentre outros. A abordagem estatística segue a seguinte ordem: (i) a análise de variância (Teste F - Snedecor); (ii) comparação das médias dos tratamentos com o controle ou testemunha (Dunnett) e (iii) em caso de interação entre os fatores, a comparação de médias (Tukey).

\section{Resultados e Discussão}

Nesta seção estão apresentados os dados relativos à resistência específica à tração obtidos do experimento. Esta variável resposta é um indicativo do gasto de energia por unidade de solo mobilizado haja vista ser a razão entre a força de tração e a área de solo mobilizada; portanto, quanto menor o valor melhor a situação. Como existem duas variáveis distintas que, relacionadas, resultam no critério de comparação, elas serão primeiramente apresentadas.

Na Tabela 4 pode-se observar, para a força horizontal média na barra de tração (FHMBT) que tanto para a menor velocidade testada quanto para a maior, houve interação significativa entre os fatores aprofundamento (Ap) e avanço (Av); além disto, o efeito dos tratamentos foi significativo e o bloqueamento mostrou-se necessário em todas as velocidades. Esta interação entre os fatores indica que existe uma dependência entre eles e

Tabela 4. Resumo da análise de variância da força horizontal média na barra de tração $\left(\mathrm{F}_{\mathrm{HMBT}}\right)$

\begin{tabular}{|c|c|c|c|c|}
\hline \multirow{2}{*}{ F.V. } & \multirow{2}{*}{ G.L. } & $V_{1}=3$ & $V_{2}=6$ & $V_{3}=7$ \\
\hline & & \multicolumn{3}{|c|}{$\left(\mathrm{km} \mathrm{h}^{-1}\right)$} \\
\hline Fator Ap & 1 & $\star \star$ & $\star \star$ & ns \\
\hline Fator Av & 2 & $\star \star$ & $\star \star$ & $\star \star$ \\
\hline Int. Ap x Av & 2 & $\star \star$ & $\mathrm{ns}$ & ** \\
\hline Fat. x Test. & 1 & ns & * & $\star \star$ \\
\hline Tratamentos & 6 & $\star \star$ & $\star \star$ & $\star \star$ \\
\hline Blocos & 3 & * & $\star \star$ & $\star \star$ \\
\hline Resíduo & 18 & & & \\
\hline Total & 27 & & & \\
\hline
\end{tabular}

Teste "F"; ** significativo a nível de $1 \%$ de probabilidade $(p<0,01) ;{ }^{*}$ significativo a nível de $5 \%$ de probabilidade $(0,01 \leq p<0,05)$; ns não significativo $(p \leq 0,05)$ que as forças médias resultantes são diferentes quando um fator varia dentro dos níveis do outro.

Quando se comparam as médias da FHMBT dos tratamentos inovadores com aquelas da sua respectiva testemunha, para cada um dos níveis de velocidade testados, pelo método de Dunnett (Tabela 5), observa-se diferença estatística em nível de cinco por cento de erro nos três casos.

Tabela 5. Comparativo da força horizontal média na barra de tração $-\mathrm{F}_{\text {НMBт }}(\mathrm{N})$ para os três níveis de velocidade $(\mathrm{V})$

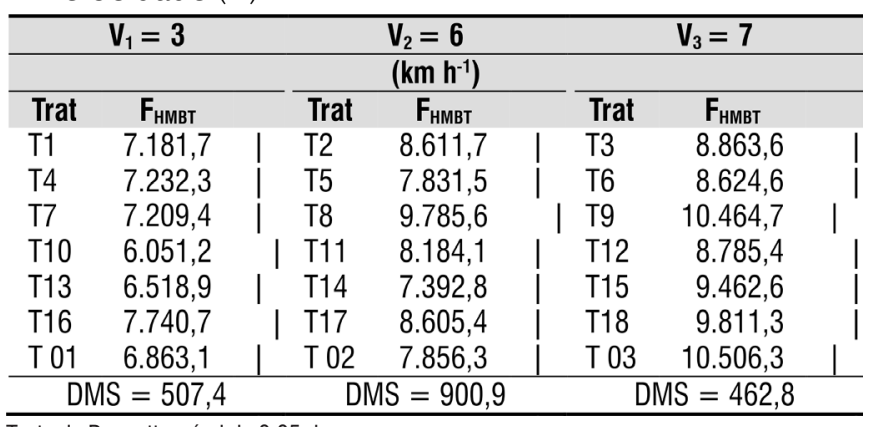

Teste de Dunnett a nível de 0,05 de erro

Na menor velocidade apenas o tratamento com rompimento em duas camadas de $15 \mathrm{~cm}$ cada uma e avanço de $40 \mathrm{~cm}$ mostrouse com menor demanda de força e diferiu significativamente da testemunha. Na velocidade intermediária observa-se que existe diferença estatística apenas entre o tratamento com três hastes e avanço de $20 \mathrm{~cm}$ (T8) e a testemunha (T02), sendo esta diferença a favor do controle. Mesmo não havendo diferença significativa entre a testemunha e os outros tratamentos, neste nível de velocidade o rompimento em três camadas mostrouse em desvantagem, tanto em referência à operação em duas camadas quanto à testemunha, considerando-se todos os níveis de avanço. Além disto, só existem dois efeitos inferiores àquele causado pelo rompimento em única camada, que ocorrem no T5 $(10 ; 30 ; 6)$ e T14 $(15 ; 30 ; 6)$, com este último, com relação a T02 (30;00;6), em torno de $6 \%$ menor. No que se refere à velocidade de $7 \mathrm{~km} \mathrm{~h}^{-1}$, conclui-se que há diferença estatística a favor de todos os tratamentos em que o rompimento do solo se dá em duas camadas: T12 (15; 40; 7), T15 $(15 ; 30 ; 7)$ e T18 $(15 ; 20 ; 7)$. Quando há ruptura com três hastes, as situações mais favoráveis ocorrem nos dois maiores avanços, isto é, em T3 $(10 ; 40 ; 7)$ e T6 $(10 ; 30 ; 7)$.

De vez que houve interação entre os fatores na menor velocidade, conforme Tabela 6 percebem-se, ao comparar o comportamento de cada patamar do aprofundamento dentro dos níveis do avanço, tendências diferentes. Na operação em três camadas simultâneas não existe diferença significativa enquanto no rompimento em duas a melhor situação é definida quando a distância entre as frentes de ruptura é $40 \mathrm{~cm}$; analisando a influência dos avanços em cada nível do aprofundamento observa-se que tanto para 40 quanto para $30 \mathrm{~cm}$ a tendência é a mesma, menor força para rompimento em duas camadas. Tal tendência se inverte para o avanço de $20 \mathrm{~cm}$.

Considerando o desdobramento dos efeitos do aprofundamento dentro de cada nível do avanço na maior 
velocidade, como mostrado na Tabela 6, verifica-se que o comportamento não apresenta a mesma tendência para a escarificação em três camadas iguais e simultâneas; os resultados alcançados foram, em ordem crescente de força, para o avanço de 30,40 e $20 \mathrm{~cm}$ enquanto a operação em duas camadas apresenta menor demanda a tração no avanço de 40 , crescendo em 30 e atingindo o máximo em $20 \mathrm{~cm}$. As amplitudes máximas de variação alcançam $1840 \mathrm{~N}$ na menor espessura de camada e $1020 \mathrm{~N}$ na maior.

Tabela 6. Médias da força horizontal média na barra de tração $-\mathrm{F}_{\text {HMBT }}(\mathrm{N})$ para $\mathrm{v}_{1}=3 \mathrm{~km} \mathrm{~h}^{-1} \mathrm{e}_{3}=7 \mathrm{~km}$ $\mathrm{h}^{-1}$ da Interação de (Ap x Av)

\begin{tabular}{cccc}
\hline $\begin{array}{c}\text { Ap } \\
\text { (cm) }\end{array}$ & $\mathbf{4 0}$ & $\mathbf{3 0}$ & $\mathbf{2 0}$ \\
& & $\mathrm{Av}(\mathrm{cm})-\mathrm{v}_{1}=3 \mathrm{~km} \mathrm{~h}^{-1}$ \\
10 & $7.181,7 \mathrm{aA}$ & $7.232,3 \mathrm{aA}$ & $7.209,4 \mathrm{bA}$ \\
15 & $6.051,2 \mathrm{bB}$ & $6.518,9 \mathrm{bB}$ & $7.740,7 \mathrm{aA}$ \\
\hline \multicolumn{3}{c}{$\mathrm{Av}(\mathrm{cm})-\mathrm{v}_{3}=7 \mathrm{~km} \mathrm{~h}^{-1}$} \\
10 & $8.863,7 \mathrm{aB}$ & $8.624,6 \mathrm{bB}$ & $10.464,7 \mathrm{aA}$ \\
15 & $8.785,4 \mathrm{aB}$ & $9.462,6 \mathrm{aA}$ & $9.811,3 \mathrm{bA}$ \\
\hline
\end{tabular}

Classificação com letras minúsculas. Linhas - Classificação com letras maiúsculas; as médias seguidas pela mesma letra não diferem estatisticamente entre si. Foi aplicado o Teste de Tukey a nível de $5 \%$ de probabilidade

Quanto aos efeitos causados pelas variações dos avanços dentro dos aprofundamentos, têm-se comportamentos diferenciados; para a maior distância entre as frentes de ruptura do solo $(40 \mathrm{~cm})$ não existe diferença estatística significativa entre as diferentes espessuras das camadas enquanto no avanço intermediário e no menor as melhores situações são, respectivamente, para o rompimento em três e duas camadas.

Tanto para a velocidade de 3 quanto para $7 \mathrm{~km} \mathrm{~h}^{-1}$, a dependência entre os fatores é semelhante, de forma que a variação dos avanços provoca mudanças mais pronunciadas nas amplitudes entre os valores de FHMBT possuindo, então, maior influência na operação. De forma geral, a FHMBT cresce com o aumento da velocidade tanto na escarificação em camada única demonstrando concordância com os resultados de Stafford (1979), Chandon \& Kushwaha (2002) e Sasaki et al. (2005), quanto naquelas em duas e três camadas simultâneas.

Os resultados relativos à área de solo mobilizada (Asm) são mostrados na Tabela 7 na qual se observa que houve interação entre os fatores aprofundamento e avanço e os efeitos dos tratamentos são significativos para os três patamares de velocidade porém o bloqueamento era necessário apenas para a velocidade de $6 \mathrm{~km} \mathrm{~h}^{-1}$.

Pela análise de Dunnett constata-se, para experimentos fatoriais diferenciados, na Tabela 8, para a menor velocidade que existe há diferença significativa entre a testemunha e os tratamentos T4 (10; 30;3) e T16 $(15 ; 20 ; 3)$, sendo o primeiro o maior valor, com área de solo mobilizada de $25,82 \%$ maior em relação à testemunha. Mesmo não diferindo estatisticamente ocorre tendência do T1 $(10 ; 40 ; 3)$ apontar para um decréscimo na área mobilizada de $9,11 \%$ em relação à testemunha. Os resultados, tanto da testemunha quanto dos demais tratamentos, foram superiores aos encontrados por Rosa et al. (2011). A
Tabela 7. Resumo da análise de variância da área de solo mobilizada (Asm)

\begin{tabular}{|c|c|c|c|c|}
\hline F.V. & G.L. & $V_{1}=3$ & $\frac{V_{2}=6}{\left(\mathrm{~km} \mathrm{~h}^{-1}\right)}$ & $V_{3}=7$ \\
\hline Fator Ap & 1 & * & ns & ns \\
\hline Fator Av & 2 & ** & ** & ns \\
\hline Int. Ap x Av & 2 & ** & ** & * \\
\hline Fat. x Test. & 1 & * & ns & * \\
\hline Tratamentos & 6 & ** & ** & * \\
\hline Blocos & 3 & ns & ** & ns \\
\hline Resíduo & 18 & & & \\
\hline Total & 27 & & & \\
\hline
\end{tabular}

Tabela 8. Comparativo da área de solo mobilizada - Asm $\left(\mathrm{m}^{2}\right)$ para os três níveis de velocidade $(\mathrm{V})-\mathrm{km} \mathrm{h}^{-1}$

\begin{tabular}{|c|c|c|c|c|c|}
\hline & $V_{1}=3$ & & $V_{2}=6$ & & $I_{3}=7$ \\
\hline \multicolumn{6}{|c|}{$\left(\mathrm{km} \mathrm{h}^{-1}\right)$} \\
\hline Trat & Asm & Trat & Asm & Trat & Asm \\
\hline T1 & 0,0721 & $\mathrm{~T} 2$ & 0,0755 & T3 & 0,0781 \\
\hline T4 & 0,0998 & T5 & 0,0960 & T6 & 0,0882 \\
\hline $\mathrm{T} 7$ & 0,0775 & T8 & 0,0821 & T9 & 0,0804 \\
\hline T10 & 0,0863 & $\mathrm{~T} 11$ & 0,0857 & T12 & 0,0834 \\
\hline T13 & 0,0847 & $\mathrm{~T} 14$ & 0,0807 & T15 & 0,0832 \\
\hline T16 & 0,0971 & $\mathrm{~T} 17$ & 0,0936 & T18 & 0,0915 \\
\hline Т 01 & 0,0793 & Т 02 & 0,0850 & T 03 & 0,0768 \\
\hline \multicolumn{2}{|c|}{ DMS $=0,0119$} & & $=0,008$ & \multicolumn{2}{|c|}{$\mathrm{DMS}=0,0112$} \\
\hline
\end{tabular}

Foi aplicado o Teste de Dunnett a nível de 0,05 de erro

velocidade de deslocamento não influenciou a Asm, constatação compartilhada por Bianchini et al. (1999).

Para a velocidade intermediária três tratamentos diferem significativamente do controle: T2 $(10 ; 40 ; 6)$, T5 $(10 ; 30 ; 6)$ e T17 $(15 ; 20 ; 6)$, sendo a melhor configuração aquela na qual a operação ocorre com rompimento em três camadas simultâneas $(\mathrm{Ap}=10 \mathrm{~cm})$ e distância longitudinal intermediária $(\mathrm{Av}=30$ $\mathrm{cm}$ ), ou seja, T5. O T2 mobiliza uma área de solo em relação à testemunha em torno de $11 \%$ menor enquanto T5 e T17 chegam próximos a 13 e $10 \%$ maiores, respectivamente. Na maior velocidade, pelo método de Dunnett, apresentado na Tabela 8, existe diferença significativa entre a testemunha e dois outros tratamentos: T6 $(10 ; 30 ; 7)$ e T18 $(15 ; 20 ; 7)$. Nos dois casos a área de solo mobilizada é maior em relação à testemunha, aproximadamente $15 \%$ para T6 e 19\% para T18.

Como existe interação significativa entre o aprofundamento e o avanço, procede-se à análise complementar na qual a Tabela 9 mostra que na menor velocidade para a operação em três camadas de $10 \mathrm{~cm}$ cada, a área mobilizada não apresenta relação direta nem inversa de proporcionalidade com o avanço enquanto no rompimento do solo em duas camadas o comportamento é inversamente proporcional com a área aumentando na medida em que a distância entre as pontas das ponteiras diminui. Quando a velocidade da operação no experimento foi de $6 \mathrm{~km} \mathrm{~h}^{-1}$, ocorreu interação significativa entre os fatores aprofundamento e avanço. É possível observar que tanto em duas quanto em três camadas não há proporcionalidade entre a área mobilizada e o avanço; contudo, as configurações com maior eficiência se repetem: $(\mathrm{Ap}=10 \mathrm{~cm}$ com $A v=30 \mathrm{~cm})$ e $(\mathrm{Ap}=15 \mathrm{~cm}$ com $\mathrm{Av}=20 \mathrm{~cm})$. 
A Tabela 9 trata da dependência entre o avanço dentro de cada nível do aprofundamento e vice-versa para todas as velocidades. $\mathrm{O}$ comportamento segue as mesmas tendências tanto de não apresentar proporcionalidade quanto das configurações com melhores resultados.

Tabela 9. Médias da área de solo mobilizada - Asm $\left(\mathrm{m}^{2}\right)$ para $\mathrm{v}_{1}=3 \mathrm{~km} \mathrm{~h}^{-1}, \mathrm{v}_{2}=6 \mathrm{~km} \mathrm{~h}^{-1} \mathrm{e}_{3}=7 \mathrm{~km} \mathrm{~h}^{-1}$ da Interação de (Ap x Av)

\begin{tabular}{cccc}
\hline $\begin{array}{c}\text { Ap } \\
\text { (cm) }\end{array}$ & $\mathbf{4 0}$ & $\mathbf{3 0}$ & $\mathbf{2 0}$ \\
& & $\mathrm{Av}(\mathrm{cm}) \mathrm{v}_{1}=3 \mathrm{~km} \mathrm{~h}^{-1}$ \\
10 & $0,0721 \mathrm{bB}$ & $0,0998 \mathrm{aA}$ & $0,0775 \mathrm{bB}$ \\
15 & $0,0863 \mathrm{aB}$ & $0,0847 \mathrm{bB}$ & $0,0971 \mathrm{aA}$ \\
\hline \multicolumn{4}{c}{$\mathrm{Av}(\mathrm{cm}) \mathrm{v}_{2}=6 \mathrm{~km} \mathrm{~h}^{-1}$} \\
10 & $0,0755 \mathrm{bB}$ & $0,0959 \mathrm{aA}$ & $0,0821 \mathrm{bB}$ \\
15 & $0,0857 \mathrm{aB}$ & $0,0807 \mathrm{bB}$ & $0,0936 \mathrm{aA}$ \\
\hline \multicolumn{3}{c}{$\mathrm{Av}(\mathrm{cm}) \mathrm{v}_{3}=7 \mathrm{~km} \mathrm{~h}^{-1}$} \\
10 & $0,0781 \mathrm{aB}$ & $0,0882 \mathrm{aA}$ & $0,0804 \mathrm{bA}$ \\
15 & $0,0833 \mathrm{aA}$ & $0,0831 \mathrm{aA}$ & $0,0914 \mathrm{aA}$ \\
\hline
\end{tabular}

Classificação com letras minúsculas. Linhas - Classificação com letras maiúsculas; as médias seguidas pela mesma letra não diferem estatisticamente entre si. Foi aplicado o Teste de Tukey ao nível de 0,05 de probabilidade

A análise de variância da resistência específica para os três níveis de velocidade apresentada na Tabela 10 evidencia a significância estatística da interação entre os fatores e o efeito dos tratamentos enquanto a necessidade de bloqueamento se faz necessária apenas para a velocidade de $6 \mathrm{~km} \mathrm{~h}^{-1}$.

Tabela 10. Resumo da análise de variância da resistência específica $\left(R_{\text {esp }}\right)$

\begin{tabular}{|c|c|c|c|c|}
\hline F.V. & G.L. & $V_{1}=3$ & $\begin{array}{c}V_{2}=6 \\
\left(\mathrm{~km} \mathrm{~h}^{-1}\right)\end{array}$ & $V_{3}=7$ \\
\hline Fator Ap & 1 & * & * & Ns \\
\hline Fator Av & 2 & ** & ** & ** \\
\hline Int. Ap x Av & 2 & $\star \star$ & ** & ** \\
\hline Fat. x Test. & 1 & ns & * & * \\
\hline Tratamentos & 6 & $\star \star$ & ** & * \\
\hline Blocos & 3 & ns & ** & Ns \\
\hline Resíduo & 18 & & & \\
\hline Total & 27 & & & \\
\hline
\end{tabular}

Através da Tabela 11 é possível observar que para a menor velocidade as configurações de hastes com melhores resultados, diferentes estatisticamente quando comparados ao controle T01 $(30 ; 00 ; 3)$, ocorrem em três tratamentos, dois com a espessura de cada camada de $15 \mathrm{~cm}$ e em um, para rompimento com aprofundamento $10 \mathrm{~cm}$, cuja ordem de classificação da menor para a maior Resistência específica é T10 (15; 40; 3), T4 (10; $30 ; 3)$ e T13 $(15 ; 30 ; 3)$. Além disto, o aumento da velocidade implicou em aumento da $\mathrm{R}_{\text {esp }}$, indo de encontro ao que AlSuhaibani \& Ghaly (2010) encontraram ao estudar a operação de um escarificador em quatro velocidades $(2,7 ; 4,3 ; 6,3$ e 8,3 $\mathrm{km} \mathrm{h}^{-1}$ ). Os mesmos autores ainda observaram que o aumento da profundidade de trabalho aumenta a variável $\mathrm{R}_{\text {esp }}$.

$\mathrm{Na}$ velocidade de $6 \mathrm{~km} \mathrm{~h}^{-1}$ três tratamentos se mostraram mais eficientes que a testemunha T02 $(30 ; 00 ; 6)$, são eles: T5 $(10 ; 30 ; 6)$, T14 $(15 ; 30 ; 6)$ e T17 $(15 ; 20 ; 6)$, em que apenas
Tabela 11. Comparativo da resistência específica $-R_{\text {es }}$ $\left(\mathrm{kN} \mathrm{m}^{-2}\right)$ para os três níveis de velocidade $(\mathrm{V})-\mathrm{km} \mathrm{h}^{-1}$

\begin{tabular}{|c|c|c|c|c|c|}
\hline & $V_{1}=3$ & & $\overline{V_{2}=6}$ & & $V_{3}=7$ \\
\hline & & & $\left(\mathrm{km} \mathrm{h}^{-}\right.$ & & \\
\hline Trat & Resp & Trat & Resp & Trat & Resp \\
\hline T1 & 99,6 & T2 & 114,1 & T3 & 113,5 \\
\hline T4 & 72,5 & T5 & 81,6 & T6 & 97,8 \\
\hline T7 & 93,0 & T8 & 119,1 & T9 & 130,1 \\
\hline T10 & 70,1 & T11 & 95,5 & T12 & 105,4 \\
\hline T13 & 77.0 & T14 & 91.6 & T15 & 113.8 \\
\hline T16 & 79,7 & T17 & 91.9 & T18 & 107.3 \\
\hline Т 01 & 86,5 & T 02 & 92,5 & Т 03 & 136,8 \\
\hline & $S=8$ & & $S=9$ & & $S=1$ \\
\hline
\end{tabular}

Foi aplicado o Teste de Dunnett a nível de 0,05 de erro

a primeira montagem apresenta diferença significativa. $\mathrm{Na}$ maior velocidade testada cinco são os tratamentos que se mostram mais eficientes com diferença significativa em relação à testemunha T03 $(30 ; 00 ; 7)$, assim classificados em ordem do menor para o maior: T6 $(10 ; 30 ; 7), \mathrm{T} 12(15 ; 40 ; 7)$, T18 $(15 ; 20$; 7), T15 (15;30;7) e T3 $(10 ; 40 ; 7)$. Observando conjuntamente os três níveis de velocidade, duas configurações se destacam entre as mais promissoras neste critério $\left(\mathrm{R}_{\text {esp }}\right)$, aparecendo em todas elas uma com rompimento em três camadas e distância entre as pontas das ponteiras de $30 \mathrm{~cm}$ e outra com aprofundamento de $15 \mathrm{~cm}$ e avanço de $30 \mathrm{~cm}$.

\section{Conclusões}

1. Em todos os níveis de velocidade existe pelo menos um resultado abaixo daquele referente ao tratamento testemunha, para $7 \mathrm{~km} \mathrm{~h}^{-1}$; os quais são, em número de cinco e em ordem crescente, T6, T12, T18, T15 e T3 enquanto se tem, no nível intermediário, apenas um $\mathrm{T} 5$ e no nível mínimo são três tratamentos T10, T4 e T13, sendo que T10 é 19\% menor que T01, T5 é $12 \%$ menor que T02 e T6 é 28\% inferior a T03.

2. As montagens das ferramentas para rompimento do solo tanto em duas camadas de $15 \mathrm{~cm}$ quanto em três de $10 \mathrm{~cm}$ e distância longitudinal entre as pontas das ponteiras tanto de 30 quanto de $40 \mathrm{~cm}$, se apresentaram com melhor desempenho em relação ao rompimento em camada única.

\section{Literatura Citada}

Al-Suhaibani, S. A.; Ghaly, A. E. Effect of plowing depth of tillage and forward speed on the performance of a medium size chisel plow operating in a sandy soil. American Journal of Agricultural and Biological Sciences, v.5, p.247-255, 2010.

Banzatto, D. A.; Kronka, S. N. Experimentação agrícola. Jaboticabal: FUNEP, 2008. 249p.

Beutler, A. N.; Centurion, J. F. Compactação do solo no desenvolvimento radicular e na produtividade da soja. Pesquisa Agropecuária Brasileira, v.39, p.581-588, 2004.

Beutler, A. N.; Centurion, J. F.; Silva, A. P.; Roque, C. G.; Ferraz, M. V. Compactação do solo e intervalo hídrico ótimo na produtividade de arroz de sequeiro. Pesquisa Agropecuária Brasileira, v.39, p.575-580, 2004. 
Bianchini A.; Marcos H. C.; Sabino, P. H. M.; Sguarezzi, B.; Sguarezzi, J. J. Comportamento operacional de um escarificador de hastes parabólicas em solo de cerrado. Revista Brasileira de Engenharia Agrícola e Ambiental, v.3, p.395-401, 1999.

Chandon, K.; Kushwaha R. L. Soil force and shank vibration on deep tillage. ASAE Annual International Meeting/World Congress Sponsored by ASAE, 15 and CIGR Hyatt Regency, 2002, Chicago. Anais... Chicago: ASAE, 2002. CD Rom

Collares, G. L.; Reinert, D. J.; Reichert, J. M.; Kaiser, D. R. Qualidade física do solo na produtividade da cultura do feijoeiro num Argissolo. Pesquisa Agropecuária Brasileira, v.41, p.1663-1674, 2006.

Drescher, M. S.; Eltz, F. L. F.; Denardin, J. E.; Faganello, A. Persistência do efeito de intervenções mecânicas para a descompactação de solos sob plantio direto. Revista Brasileira de Ciência do Solo, v.35, p.713-1722, 2011.

EMBRAPA - Empresa Brasileira de Pesquisa Agropecuária. Manual de métodos de análise de solo. Centro Nacional de Pesquisa de Solos. 2.ed., Rio de Janeiro: EMBRAPA, 1997. 212p.

Flowers, M. D.; Lal, R. Axial load and tillage effects on soil physical properties and soybean grain yield on a molic ochraqualf in northwest Ohio. Soil and Tillage Research, v.48, p.21-35, 1998.

Foloni, J. S. S.; Calonego, J. C.; Lima, S. L. Efeito da compactação do solo no desenvolvimento aéreo e radicular de cultivares de milho. Pesquisa Agropecuária Brasileira, v.38, p.947953, 2003.

Godwin, R. J. A review of the effect of implement geometryon soil failure and implement forces. Soil \& Tillage Research, v.97, p.331-340, 2007.
Machado, A. L. T.; Reis, A. V.; Moraes, M. L. B.; Alonço, A. dos S. Máquinas para preparo do solo, semeadura, adubação e tratamentos culturais. 2.ed. Pelotas: UFPEL, 2005. 253p.

Nicoloso, S. N.; Amado, T. J. C.; Schneider, S.; Lanzanova, M. E.; Giraderllo, V. C.; Bragagnolo, J. Eficiência da escarificação mecânica e biológica na melhoria dos atributos físicos de um Latossolo muito argiloso e no incremento do Rendimento de soja. Revista Brasileira de Ciência do Solo, v.32, p.17231734, 2008.

Rosa, D. P.; Reichert, J. M.; Mentges, M. I.; Barros, C. A. P.; Reinert, D. J.; Vieira, D. A. Cultivo mínimo: Efeito da compactação e deformação abaixo da atuação da ponteira do subsolador. Revista Brasileira Engenharia Agrícola Ambiental, v.15, p.1199-1205, 2011.

Russini, A. Projeto, construção e teste de Instrumentação eletrônica para avaliação do desempenho de tratores agrícolas. Santa Maria: UFSM 2009. 142p. Dissertação Mestrado

Sasaki, C. M.; Gonçalvez J. L. M.; Bentivenha, S. R. P. Desempenho operacional de hastes subsoladoras em função da ponteira e do tipo do solo. Scientia Forestalis, v.67, p.4452, 2005.

Silva, F. de A. S. e; Azevedo, C. A. V. de. Versão do programa computacional Assistat para o sistema operacional windows. Revista Brasileira de Produtos Agroindustriais, v.4, p.71-78, 2002.

Soane, B. D.; Ouwerkerk, C. van. Soil compaction problems in world agriculture. In: Soane, B. D.; Ouwerkerk, C. van. (ed.) Soil compaction in crop production. Netherlands: Elsevier, 1994. 21p.

Stafford, J. V. The performance of a rigid tine in relation to soil properties and speed. Journal of Agricultural Engineering Research, v.24, p.41-55, 1979. 